\title{
Social Jetlag is Independently Associated with Chronotype and Poor Memory for Extinguished Fear
}

\author{
Ryan Bottary ${ }^{1,2,3, *}$ (D), Tony J. Cunningham ${ }^{3,4,5}$, Rebecca M.C. Spencer ${ }^{6}$ and \\ Edward F. Pace-Schott ${ }^{1,2,4,6}$ \\ ${ }^{1}$ Department of Psychiatry, Massachusetts General Hospital, Charlestown, MA, USA, ${ }^{2}$ Athinoula A. Martinos Center for \\ Biomedical Imaging, Charlestown, MA, USA, ${ }^{3}$ Department of Psychology and Neuroscience, Boston College, Chestnut Hill, \\ MA, USA, ${ }^{4}$ Department of Psychiatry, Harvard Medical School, Boston, MA, USA, ${ }^{5}$ Department of Psychiatry, Beth Israel \\ Deaconess Medical Center, Boston, MA, USA, and ${ }^{6}$ Department of Psychological and Brain Sciences, University of \\ Massachusetts Amherst, Amherst, MA, USA \\ ${ }^{*}$ Corresponding author: Email: ryan.bottary@bc.edu \\ Research was conducted in MGH psychiatry and Martino's center.
}

(Received 30 January 2020; Revised 16 May 2020; Accepted 02 June 2020)

\begin{abstract}
Social jetlag (SJ) occurs when sleep-timing irregularities from social or occupational demands conflict with endogenous sleep-wake rhythms. SJ is associated with evening chronotype and poor mental health, but mechanisms supporting this link remain unknown. Impaired ability to retrieve extinction memory is an emotion regulatory deficit observed in some psychiatric illnesses. Thus, SJ-dependent extinction memory deficits may provide a mechanism for poor mental health. To test this, healthy male college students completed 7-9 nights of actigraphy, sleep questionnaires, and a fear conditioning and extinction protocol. As expected, greater SJ, but not total sleep time discrepancy, was associated with poorer extinction memory. Unexpectedly, greater SJ was associated with a tendency toward morning rather than evening chronotype. These findings suggest that deficient extinction memory represents a potential mechanism linking SJ to psychopathology and that $\mathrm{SJ}$ is particularly problematic for college students with a greater tendency toward a morning chronotype.
\end{abstract}

Keywords: Social Jetlag; Chronotype; Fear Extinction; Emotional Memory; Sleep

\section{Introduction}

Fear extinction memory (i.e. remembering that something that once signaled danger no longer does so) is a form of emotion regulation that is strengthened by sleep (Pace-Schott, Germain et al., 2015), but impaired in individuals with certain psychiatric illnesses such as post-traumatic stress disorder, obsessive compulsive disorder and schizophrenia (Milad \& Quirk, 2012). In addition to poor emotion regulation, psychopathology is often accompanied by poor sleep quality and is associated with evening chronotype (i.e. delayed bed- and rise-times) (Antypa et al., 2016) and sleep-pattern disruptions (Foster et al., 2013). Social jetlag (SJ) is a form of desynchrony between endogenous sleep-wake rhythms and actual sleepwake timing imposed by social or occupational demands (Wittman et al., 2006). SJ is expressed as delayed sleep timing (i.e. later sleep midpoint) on weekends compared to weekdays. SJ is linked to psychopathology (Foster et al., 2013; Levandovski et al., 2011), but mechanisms linking the two remain poorly understood.

\footnotetext{
(c) The Author(s), 2020. Published by Cambridge University Press. This is an Open Access article, distributed under the terms of the Creative Commons Attribution licence (http://creativecommons.org/licenses/by/4.0/), which permits unrestricted re-use, distribution, and reproduction in any medium, provided the original work is properly cited.
} 


\section{Objective}

In this secondary analysis of a larger study (Pace-Schott et al., 2013; Pace-Schott, Rubin et al., 2015), we extend our findings by measuring the association between SJ and extinction memory. Although sleep deprivation disrupts extinction memory (Pace-Schott, Germain et al., 2015), little work has addressed the effects of sleep timing irregularities on retention of extinction memory. Here, we hypothesized that SJ represents a form of sleep-timing irregularity that might impair extinction memory, thus resulting in increased risk for psychopathology.

\section{Methods}

Seventy-six healthy male college students (aged 18-29) produced 7-9 nights of wrist actigraphy then completed a 2-session Fear Conditioning, Extinction Learning and Extinction Recall protocol (see Supplementary Materials for additional methodological details). SJ was determined by subtracting average weekday from weekend actigraphy-determined sleep midpoint (see Supplementary Materials for days included in averages and Table S1 for weekday and weekend mean sleep timing information). This calculation was then repeated for average total sleep time (TST). During Conditioning (occurring in the morning or evening) a finger-shock established anticipatory skin-conductance responses (SCR) to 2 differently colored lamps (CS+) but not a third (CS-). One CS+ (CS + E) but not the other $(\mathrm{CS}+\mathrm{U})$ was then immediately extinguished during Extinction. During Recall (3-, 12- or 24-hr after Extinction), all 3 CS were presented. Extinction memory was calculated separately for SCR (ERI) and differential SCR (dERI). The authors assert that all procedures contributing to this work comply with the ethical standards of the relevant national and institutional committees on human experimentation and with the Helsinki Declaration of 1975, as revised in 2008.

\section{Results}

As predicted, SJ was negatively associated with ERI $(\mathrm{r}=-.248, \mathrm{p}=.035)$ and dERI $(\mathrm{r}=-.280, \mathrm{p}=.018)$ (See Figure 1). These associations were not driven by time-of-day of testing or recall delay and SJ did not correlate with non-extinction memory-related reactivity (see Supplementary Materials). Greater SJ was associated with higher Morningness-Eveningness Questionnaire (MEQ) scores (Horne \& Ostberg, 1976; $\mathrm{r}=.31, \mathrm{p}=.007)$. Note, higher MEQ score indicates greater morningness, though only 2 participants qualified as moderate morning types (score between 59-69) and 1 as a definite morning type (score $>69$ ) based on MEQ scoring criteria. MEQ was not correlated with ERI $(\mathrm{r}=-.13, \mathrm{p}=.27)$ or dERI $(\mathrm{r}=-.12$, $\mathrm{p}=.31$ ). Lastly, difference in average weekend and weekday total sleep time was not associated with ERI $(\mathrm{r}=.03, \mathrm{p}=.80)$ or dERI $(\mathrm{r}=.001, \mathrm{p}=.99)$, suggesting the greater importance of weekday/weekend sleep timing differences versus weekday/weekend sleep-duration discrepancies.

\section{Discussion}

The present findings indicate that greater SJ is associated with poorer extinction memory, revealing one potential link between SJ and psychopathology. Higher SJ was also associated with greater morningness (See Figure 2), an observation that contrasts with previous reports that suggest evening types are more likely to experience SJ (e.g. Foster et al., 2013). That greater morningness did not directly correlate with extinction memory remains puzzling. Further, other studies have linked greater eveningness, rather than morningness, to impaired extinction memory-related processing (i.e. extinction generalization; PaceSchott, Rubin et al., 2015) and psychopathology like depression (Levandovski et al., 2011) and anxiety (Lemoine et al., 2013). However, at least one study has linked morningness in males to greater neuroticanxious personality traits (Muro et al., 2009) and morning-type college students report lower ratings of psychological quality of life compared with intermediate- and evening-type students (Lau et al., 2013). Late-night social schedules, especially on weekends, may disproportionately affect morning types by 


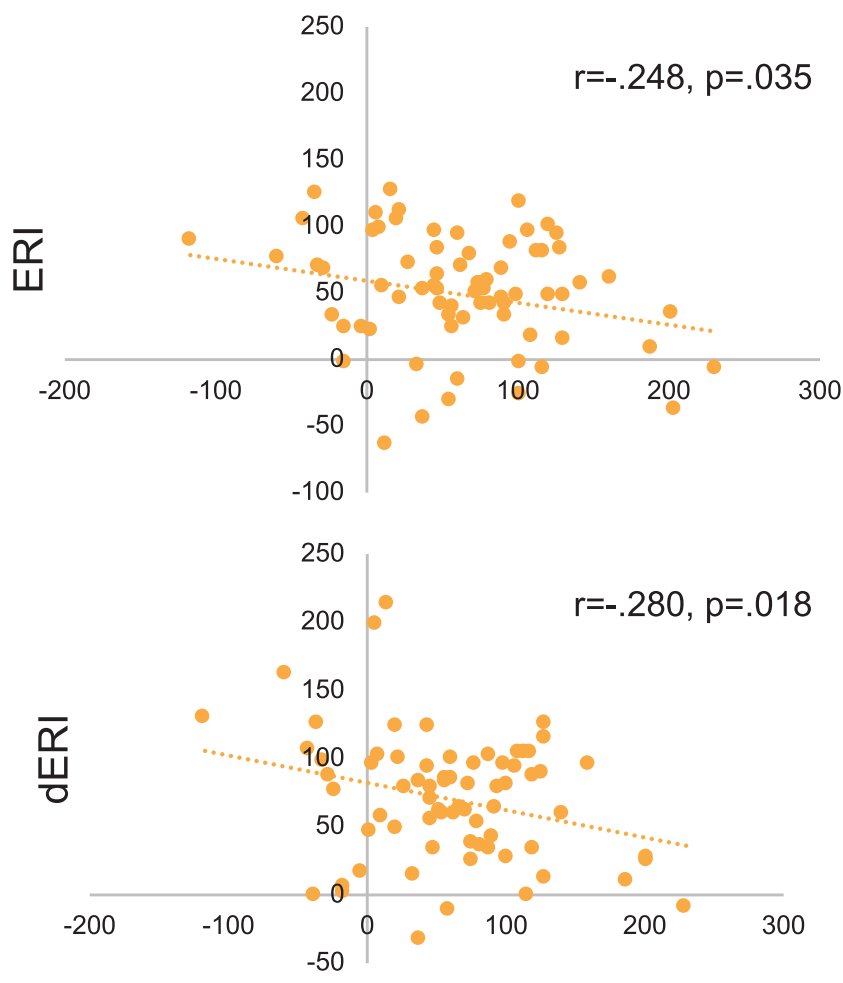

Social Jetlag

Figure 1. Association between social jetlag (determined by subtracting weekday from weekend mean actigraphy-measured sleep midpoint expressed as minutes past midnight) and extinction memory determined by (a) Extinction Retention Index (ERI) and (b) differential ERI (dERI; corrects for general increases in reactivity) (see Supplementary Materials for ERI/dERI calculations). Note better memory for extinction is expressed as greater ERI or dERI.

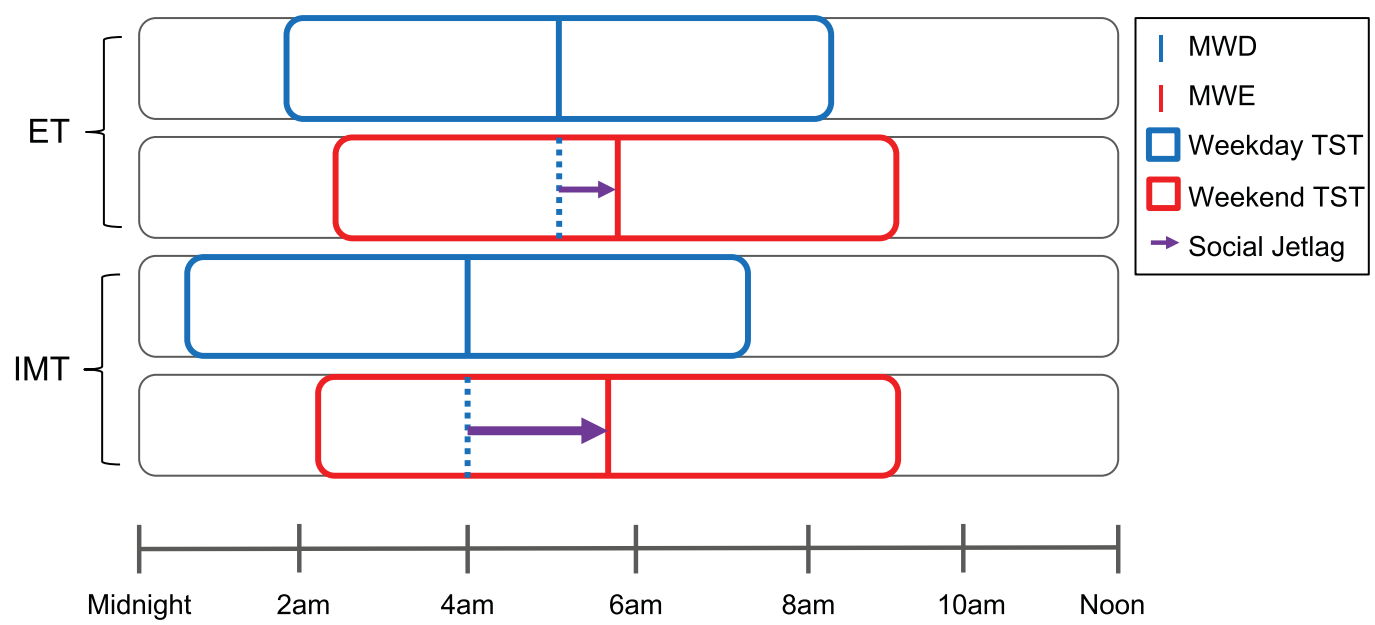

Figure 2. Sleep timing on weekdays and weekends for evening types (ET) and intermediate-morning/morning types (IMT). Social jetlag (i.e. later sleep midpoint on weekends compared to weekdays) was greater in those with greater tendency toward morningness based on Morningness-Eveningness Questionnaire (MEQ; Horne and Ostberg, 1976) scores. Here, ET reflects averages across true evening types while IMT reflects averages across the upper half (higher MEQ scores, based on a median split) of intermediate types (Pace-Schott et al., 2015). This split included 2 moderate morning types and 1 definite morning type based on Horne and Ostberg (1976) scoring criteria. Note. MWD = average sleep midpoint on weekday nights; $\mathrm{MWE}=$ average sleep midpoint on weekend nights; TST = total sleep time. 
generating greater discrepancy between bedtimes on weekends and weekdays compared with evening types. Social jetlag, in turn, impaired emotion processing (i.e. extinction memory) and thus may be a vulnerability factor for poor mental health outcomes in those with morning chronotypes. Further, SJ appears to directly impact emotion processing rather than simply being a consequence of pre-existing psychopathology and late sleep timing, though further work is needed to confirm this.

\section{Conclusions}

Taken together, our observations suggest that, in young-adult male college students, greater morningness is a potential risk factor for SJ, which in turn is associated with impaired extinction memory. As both SJ and extinction memory deficits have been linked to poor mental health, SJ, expressed as delayed sleep timing on weekends compared to weekdays, may represent a particularly harmful sleeping pattern for optimal emotion regulation. Further, deficiencies in extinction memory may represent a specific mechanism linking SJ to poor mental health. Given that greater morningness was also associated with SJ, adopting greater coherence between weekend and weekday sleep timing may be particularly important for morning-type college students.

Acknowledgements. Special thanks to Dr. Zoe S. Rubin, MD for her assistance with data processing and analysis.

Author Contributions. EFPS and RMCS conceived and designed the study. EFPS conducted data gathering. RB and EFPS devised the secondary analysis plan and performed statistical analyses. RB, TJC, and EFPS drafted and revised the manuscript.

Financial Support. This work was supported by the National Institutes of Mental Health (NIMH R21MH090357) and the National Institute on Aging (NIA R00AG029710).

Data Availability Statement. Readers can contact the authors if they wish to access relevant materials for replication. Detailed protocols are available in the Supplementary Materials and in Pace-Schott et al. (2013) and Pace-Schott, Rubin et al. (2015).

Conflict of Interest. The authors have no relevant conflicts of interest to report.

Supplementary Materials. To view supplementary material for this article, please visit http://dx.doi.org/10.1017/exp.2020.26.

\section{References}

Antypa, N., Vogelzangs, N., Meesters, Y., Schoevers, R., \& Penninx, B. W. (2016). Chronotype associations with depression and anxiety disorders in a large cohort study. Depression and Anxiety, 33, 75-83.

Foster, R. G., Peirson, S. N., Wulff, K., Winnebeck, E., Vetter, C., \& Roenneberg, T. (2013). Sleep and circadian rhythm disruption in social jetlag and mental illness. Progress in Molecular Biology and Translational Science, 119, $325-346$.

Horne, J. A., \& Östberg, O. (1976). A self-assessment questionnaire to determine morningness-eveningness in human circadian rhythms. International Journal of Chronobiology, 4, 97-110.

Lau, E. Y. Y., Wong, M. L., Ng, E. C. W., Hui, C. C. H., Cheung, S. F., \& Mok, D. S. Y. (2013). “Social jetlag” in morning-type college students living on campus: Implications for physical and psychological well-being. Chronobiology International, 30, 910-918.

Lemoine, P., Zawieja, P., \& Ohayon, M. M. (2013). Associations between morningness/eveningness and psychopathology: An epidemiological survey in three in-patient psychiatric clinics. Journal of Psychiatric Research, 47, 1095-1098.

Levandovski, R., Dantas, G., Fernandes, L. C., Caumo, W., Torres, I., Roenneberg, T., \& Allebrandt, K. V. (2011). Depression scores associate with chronotype and social jetlag in a rural population. Chronobiology International, 28, 771-778.

Milad, M. R., \& Quirk, G. J. (2012). Fear extinction as a model for translational neuroscience: Ten years of progress. Annual Review of Psychology, 63, 129-151.

Muro, A., Gomà-i-Freixanet, M., \& Adan, A. (2009). Morningness-eveningness, sex, and the alternative five factor model of personality. Chronobiology International, 26, 1235-1248.

Pace-Schott, E. F., Germain, A., \& Milad, M. R. (2015). Effects of sleep on memory for conditioned fear and fear extinction. Psychological Bulletin, 141, 835.

Pace-Schott, E. F., Rubin, Z. S., Tracy, L. E., Spencer, R. M., Orr, S. P., \& Verga, P. W. (2015). Emotional trait and memory associates of sleep timing and quality. Psychiatry Research, 229, 999-1010. 
Pace-Schott, E. F., Spencer, R. M., Vijayakumar, S., Ahmed, N. A., Verga, P. W., Orr, S. P., \& Milad, M. R. (2013). Extinction of conditioned fear is better learned and recalled in the morning than in the evening. Journal of Psychiatric Research, 47, $1776-1784$.

Wittmann, M., Dinich, J., Merrow, M., \& Roenneberg, T. (2006). Social jetlag: Misalignment of biological and social time. Chronobiology international, 23, 497-509.

Cite this article: Bottary R, Cunningham TJ, Spencer RMC, Pace-Schott EF (2020). Social Jetlag is Independently Associated with Chronotype and Poor Memory for Extinguished Fear Experimental Results, 1, e22, 1-8. https://doi.org/10.1017/ exp. 2020.26 


\title{
Peer Reviews
}

\author{
Reviewing editor: Dr. Jessica Payne \\ University of Notre Dame, Notre Dame, Indiana, United States, 46556
}

This article has been accepted because it is deemed to be scientifically sound, has the correct controls, has appropriate methodology and is statistically valid, and met required revisions.

doi:10.1017/exp.2020.26.pr1

\section{Review 1: Social Jetlag is Independently Associated with Chronotype and Poor Memory for Extinguished Fear}

Reviewer: Brant Hasler

Date of review: 16 March 2020

Published online:

(C) The Author(s), 2020. Published by Cambridge University Press This is an Open Access article, distributed under the terms of the Creative Commons Attribution licence (http://creativecommons.org/licenses/by/4.0/), which permits unrestricted re-use, distribution, and reproduction in any medium, provided the original work is properly cited.

Conflict of interest statement. Reviewer declares none

Comments to the Author: In this manuscript, the authors examined whether social jet lag was associated with poorer extinction memory and/or evening chronotype in a sample of healthy male college students. This is a novel and interesting question, with relevance to understanding oft-noted associations between eveningness and worse psychological health. Although college students are arguably not the ideal sample for examining social jet lag as traditionally defined (see below), the manuscript could still provide a worthwhile contribution to the literature. I suggest considering the following comments/concerns:

1) More detail about weekday and weekend actigraphy data would be useful, including providing the \# of weekend days available (with 9 days of data, some individuals may have 2 sets of weekend data), what weekend data was included in the means if more than one weekend was available, and the separate mean sleep timing data for both weekdays and weekends.

2) Relatedly, college students are less bound to the traditional weekday/weekend schedules (e.g., Thursday is often treated as a weekend night in terms of social activities and sleep schedule), and also have less systematically imposed early start times on weekdays (due to relatively greater control over their class schedules), raising questions about the meaningfulness of the traditional "social jet lag" definition. This may be relevant to the unexpected finding that morning chronotypes exhibited greater social jet lag in this sample (although the authors' speculation that college schedules may be more difficult for morning-types is worth consideration as well).

3) Notably, the authors' seemingly ignore that social jet lag in the opposite direction (advancing on the weekend) is seemingly associated with improved extinction memory. Other social jet lag papers have also ignored this issue, but it deserves discussion. Why would relative delay on weekdays be less problematic than relative delay on the weekend? In the more typical cases where eveningness and social jet lag are associated, one could argue that a delayed state is more adaptive, and thus spending relatively less time in the advanced state on weekends is a good thing. But that explanation would not seem to fit the current circumstances, when morningness was associated with greater social jet lag. 
4) The authors could do more to address the lack of association between chronotype (as measured by MEQ) and extinction memory, especially given that social jet lag (which did correlate with extinction memory) was associated with morningness, which should be associated with better extinction memory based on consistent findings that eveningness is often accompanied by psychological/physical dysfunction. I wondered if it would be worth looking at whether the chronotype-extinction memory association is moderated by social jet lag-that is, in the morning-types with the most social jet lag, is there also worse extinction memory?

5) Finally, the authors could better distinguish between the standard SCR and differential SCR measures - they have the room in the Supplemental section, and it's currently unclear how the literature views these two different approaches (whether one is more valid in certain circumstances, etc.).

\section{Score Card}

Presentation

3.9

Is the article written in clear and proper English? (30\%)

Is the data presented in the most useful manner? (40\%)

Does the paper cite relevant and related articles appropriately? (30\%)

\section{Context}

Does the title suitably represent the article? (25\%)

Does the abstract correctly embody the content of the article? (25\%)

Does the introduction give appropriate context? (25\%)

Is the objective of the experiment clearly defined? (25\%)

Analysis

Does the discussion adequately interpret the results presented? (40\%)

Is the conclusion consistent with the results and discussion? (40\%)

Are the limitations of the experiment as well as the contributions of the experiment clearly outlined? (20\%) 


\section{Review 2: Social Jetlag is Independently Associated with Chronotype and Poor Memory for Extinguished Fear}

Reviewer: Dr. Daniel Denis

Date of review: 13 April 2020

Published online:

(c) The Author(s), 2020. Published by Cambridge University Press This is an Open Access article, distributed under the terms of the Creative Commons Attribution licence (http://creativecommons.org/licenses/by/4.0/), which permits unrestricted re-use, distribution, and reproduction in any medium, provided the original work is properly cited.

Conflict of interest statement. Reviewer declares none.

Comments to the Author: The authors present data showing that social jetlag in college students is positivelty associated with greater morningness and is negatively associated with fear extinction memory. The paper is well written with appropriate analyses. The results highlight the negative consequences of social jetlag on a specific domain of cognitive functioning. The authors should clarify whether the result reported on lines 90-91 ("SJ was positively associated with Recall CS+E reactivity (i.e. lower ERI/dERI) ...") is referring back to the result reported in the Figure, or is a separate analysis. If it is a separate analysis, results of the statistical test should be reported.

\section{Score Card}

Presentation

5.0

Is the article written in clear and proper English? (30\%)

Is the data presented in the most useful manner? (40\%)

Does the paper cite relevant and related articles appropriately? (30\%)

Context

5.0

Does the title suitably represent the article? (25\%)

Does the abstract correctly embody the content of the article? (25\%)

Does the introduction give appropriate context? (25\%)

Is the objective of the experiment clearly defined? (25\%)

Analysis

Does the discussion adequately interpret the results presented? (40\%)

Is the conclusion consistent with the results and discussion? (40\%)

Are the limitations of the experiment as well as the contributions of the experiment clearly outlined? $(20 \%)$ 